\title{
LE SOLUTRÉEN ANCIEN EN ARDĖCHE (FRANCE): UNE RÉVISION CRITIQUE DES INDUSTRIES LITHIQUES
}

\section{The early Solutrean in Ardeche (France): a reappraisal of the lithique assemblages}

\author{
Sophie Guégan ${ }^{1}$
}

Recibido el 23 de marzo de 2013. Aceptado el 26 de julio de 2013

Résumé. S'inscrivant dans un travail de Doctorat sur les peuplements des deux rives de la vallée du Rhône au Maximum Glaciaire, cet article propose un état des connaissances sur la phase ancienne du Solutréen en Ardèche (rive droite du Rhône), donnant lieu à une révision critique des industries lithiques s'y rapportant. En effet, depuis les travaux de P.E.L. Smith et J. Combier, et plus récemment le travail d'A. Barbiero consacré aux pointes à face plance et pointes à cran d'une collection ancienne à la Baume d'Oullins (GardArdèche, France), aucun réexamen global n'avait été entrepris. Trois gisements de référence pour cette phase ancienne, ont ainsi été sélectionnés: les grottes d'Oullins (Gard-Ardèche, France), de Chabot (Gard, France) et de Granouly (Ardèche, France). Nous présentons ici les réslutats préliminaires à cette étude. En premier lieu, une approche typologique a été menée principalement autour de la pointe à face plane, objet emblématique de cette phase, permettant d'en préciser les principaux caractères. Dans un deuxième temps, une approche technologique a permis de confirmer l'homogénéité dans les choix techniques des Solutréens entre l'Ardèche et les régions du Sud-Ouest et du Bassin Parisien. Enfin, une étude des matières premières, a révélé pour l'heure des choix économiques étonnants pour le contexte paléo-climatique, avec des déplacements vers le nords et un franchissement du Rhône.

Mots clés: vallée de l'Ardèche, Solutréen ancien, technologie lithique, pointes à face plane.

Abstract. In the frame of a doctoral dissertation on the Late Glacial Maximum occupations of both sides of the Rhône valley, this paper presents a state of the art and a critical reappraisal of the lithic assemblages from the early phases of the Solutrean in Ardeche (right bank of the Rhône river). Indeed, since the works of P.E.L. Smith and J. Combier, except the work of A. Barbiero, on the unifacial leaf-points and shouldered points from Baume d'Oullins, no complete study of these assemblages have been conducted. Three key-sites of this early phase have been tackled: Oullins cave (Gard-Ardèche), Chabot cave (Gard) and Granouly cave (Ardèche). Here we present the preliminary results of this new study. A typological approach have been first conducted, especially to precise the main features of the unifacial leaf-points, the most characteristic artefact of this period. Then a technological approach permited to confirm the homogeneity of the technical choices made by early Solutrean populations of Ardèche, South-Western France and Paris Basin. Finally, a study of the lithic raw material shows surprising economical choices, notably material coming from northern regions and from the other side of the Rhône valley.

Keywords: Ardèche valley, Early Solutrean, lithic technology, unifacial point.

(') UMR 5140 CNRS Laboratoire "Archéologie des sociétés méditeranéennes" et Labex ARCHIMEDE, programme "Investissement d'Avenir " ANR-11-LABX-0032-0 . Université de Montpellier III - Paul Valéry. 


\section{INTRODUCTION}

Le territoire rhodanien, richement peuplé durant tout le Paléolithique, abrite sur sa rive droite, et plus spécifiquement dans le département de l'Ardèche, une dizaine de gisements archéologiques attribués au Solutréen.

Mais tandis que la phase récente du Solutréen, dans cette région, a fait l'objet de nombreux travaux et publications (entre autres, Bazile et Bazile-Robert 1979; Boccaccio 2005), la phase ancienne à pointe à face plane quant à elle, n'avait fait l'objet d'aucune révision critique depuis les thèses de P.E.L. Smith (Smith 1966) et J. Combier (Combier 1967). Plus récemment toutefois, en 2002, un travail de maîtrise a porté sur l'analyse typologique des fossiles directeurs que sont les pointes à face plane et pointes à cran issues des anciennes fouilles à la baume d'Oullins (Gard-Ardèche, France) (Barbiero 2002), principal gisement paléolithique de la région.

Cet article s'inscrit dans un travail de doctorat en cours, consacré à l'étude des industries lithiques du Pléniglaciaire supérieur en vallée du Rhône, occupé sur la rive droite du fleuve par les Solutréens, et sur sa rive gauche, par les Epigravettiens. Nous proposons ici, un état des connaissances sur la phase ancienne du Solutréen en vallée du Rhône, donnant lieu à une révision critique des collections lihtiques disponibles, à savoir celles issues des grottes d'Oullins, (Grad-Ardèche, France), de Chabot (Gard, France) et de Granouly (Ardèche, France). Notre approche est principalement fondée sur l'étude des comportements techniques et moyens de subsistance à travers l'analyse typo-technologique du matériel lithique, couplée à une étude des matières premières.

\section{PRÉSENTATION GENERALE}

\subsection{Contexte géographique}

Le département de l'Ardèche, encaissé entre les contreforts du Massif Central au Nord-Ouest, le Rhône à l'Est et les plateaux calcaires au Sud, est un territoire naturel contrasté, riche en gisements paléolithiques (Fig. 1). L'essentiel de ces gisements se situe d'ailleurs dans le sud du département, dans les gorges de l'Ardèche. Ces gorges, creusées sous les plateaux calcaires de faciès récifal urgonien, forment un immense réseau karstique (Combier 1967) de Vallon Pontd'Arc en amont, jusqu'à Saint-Martin d'Ardèche en aval, sur près d'une trentaine de kilomètres environ. Plus d'une trentaine de gisements paléolithiques y sont référencés, dont onze se rapportent au Solutréen (Combier 1967; Gély 2008; Prud'Homme 2008).

Parmi les onze gisements solutréens, dix se situent dans les gorges. Il s'agit, d'amont en aval, de la grotte de Mézelet, de la Tête-de-Lion, des grottes n. ${ }^{\circ} 47$, et d'Oullins, de la Baou

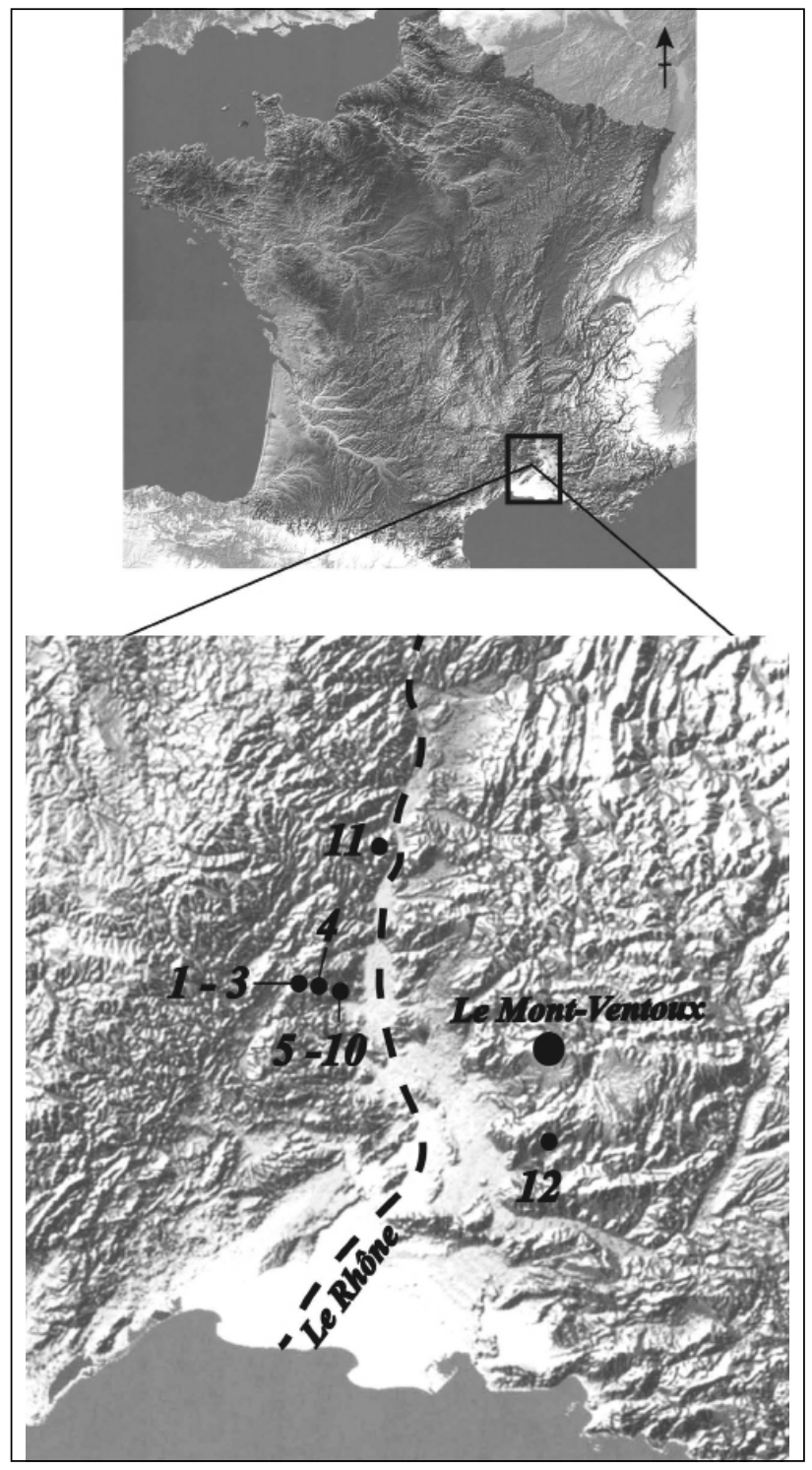

$\Delta$ Figure 1. Localisation des gisements solutréens ardéchois et épigravettien cités dans le texte. 1 - 3: Grottes de Mézelet, de la Tête du Lion et grotte n. ${ }^{\circ} 47 ; 4$ : Grotte d'Oullins; 5 - 10: Baou de la Cello, grottes Chabot, du Figuier, des Deux-Ouvertures, Huchard et Sombre; 12: La Font-Pourquière.

de la Cello, des grottes Chabot, du Figuier, des Deux-Ouvertures, Huchard et Sombre (Bazile 1990; Combier 1967 et 1973; Gély 2008; Prud'Homme 2008). Toutefois, précisons que les grottes de Mézelet, de la Tête-de-Lion et Huchard ne contiennent que des témoignages artistiques, tandis que les grottes de la Baou de la Cello et la grotte $n .^{\circ} 47$ ne contiennent, quant à elles, que des restes lithiques et osseux (Combier 1967 et 1973; Gély 2008; Prud'Homme 2008). Toutes les autres grottes ont, associés, de l'art pariétal et des restes lithiques et osseux attribués au Solutréen. Enfin, le onzième site, la grotte de Granouly, se trouve en dehors des gorges, à une quarantaine de kilomètres au nord, et une centaine de mètre du Rhône, faisant de ce gisement, le plus oriental de la sphère solutréenne (Combier 1973). 


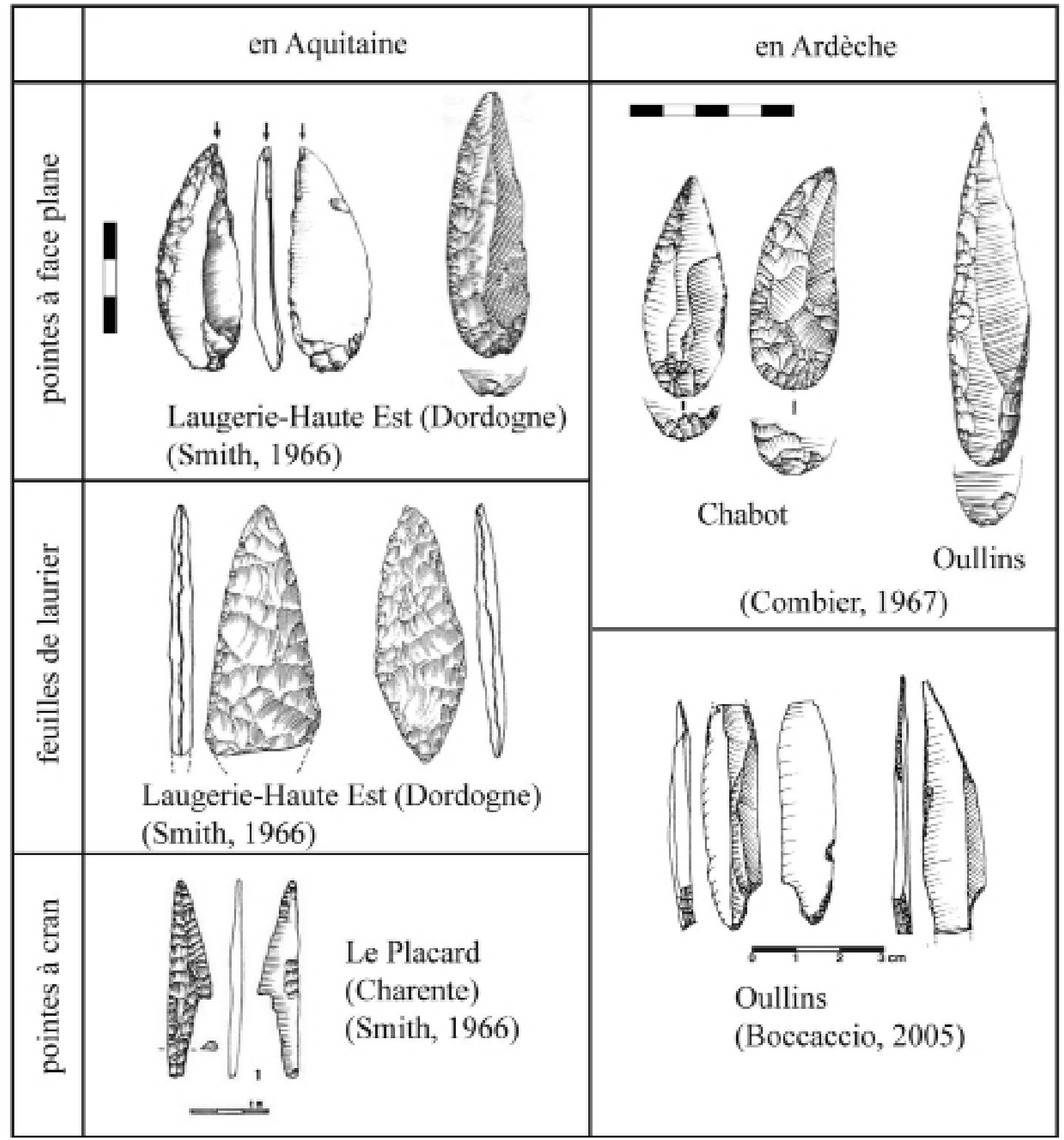

\ Figure 2. Découpage chrono-culturel du Solutréen en Aquitaine et en Ardèche.

\subsection{Rappel historiographique}

Le découpage chrono-culturel du Solutréen, fait sur la base des stratigraphies d'Aquitaine, et principalement sur celles de Laugerie-Haute Est et Ouest, dès les débuts du $X X^{e}$ siècle, demeure encore aujourd'hui à peu près le même (Smith 1966; Demars 1995; Renard 2010) et a été largement employé pour les autres régions, dont le Sud-Est de la France (Combier 1967; Bazile 1990). II se découpe ainsi en trois phases: une phase ancienne, pendant laquelle la pointe à face plane est l'unique fossile directeur, une phase moyenne qui voit l'arrivée des feuilles de laurier et durant laquelle la pointe à face plane perdure, et enfin une phase récente, où la pointe à cran fait son apparition, aux côtés de la feuille de laurier et de la pointe à face plane, qui persistent (Smith 1966). Si ce découpage a été utilisé pour l'ensemble des régions où le Solutréen est attesté, il n'en demeure pas moins presque exclusivement réservé au Sud-Ouest de la France, puisqu'en effet, pour ce qui est de la vallée du Rhône et de l'Ardèche en particulier, les strati- 
graphies des gisements les plus riches n'ont livré que deux phases : une phase ancienne à uniques pointes à face plane et une phase récente à pointes à face plane et pointes à cran méditerranéennes (Fig. 2). La phase moyenne à feuille de laurier, qui a fait la réputation du Solutréen, et qui est si largement répandue du Bassin Parisien jusqu'en péninsule lbérique, n'est présente en Ardèche, qu'au travers de quelques pièces à retouche bifaciale seulement (Combier 1967; Bazile 1990; Boccaccio 2005) tandis qu'en Languedoc, à la grotte de la Salpêtrière, semble exister un véritable niveau du Solutréen moyen à feuille de Laurier (Escalon de Fonton et Bonifay 1957), dont l'on retrouve par ailleurs des éclats de façonnage dans les niveaux sus-jacents du Salpêtrien (Boccaccio 2005).

\subsection{Chronologie}

A l'heure actuelle, les seules datations dont nous disposons pour les niveaux solutréens de cette région, sont d'une grande imprécision (Fig. 3) (Bazile 1990). Elles sont en effet, ou trop jeunes pour la phase ancienne à pointe à face plane, ou trop vieilles pour la phase récente à pointe à cran méditerranéenne, ou bien encore possèdent un écarttype trop important entraînant des recouvrements entre les phases, ce qui pour le Solutréen, qui s'étale sur deux mille ans environ, est un problème. A cela, s'ajoute un contexte chrono-stratigraphique incertain, lié à l'ancienneté des fouilles pour certains niveaux datés. La publication prochaine des dates obtenues dans le cadre du PCR "Datations

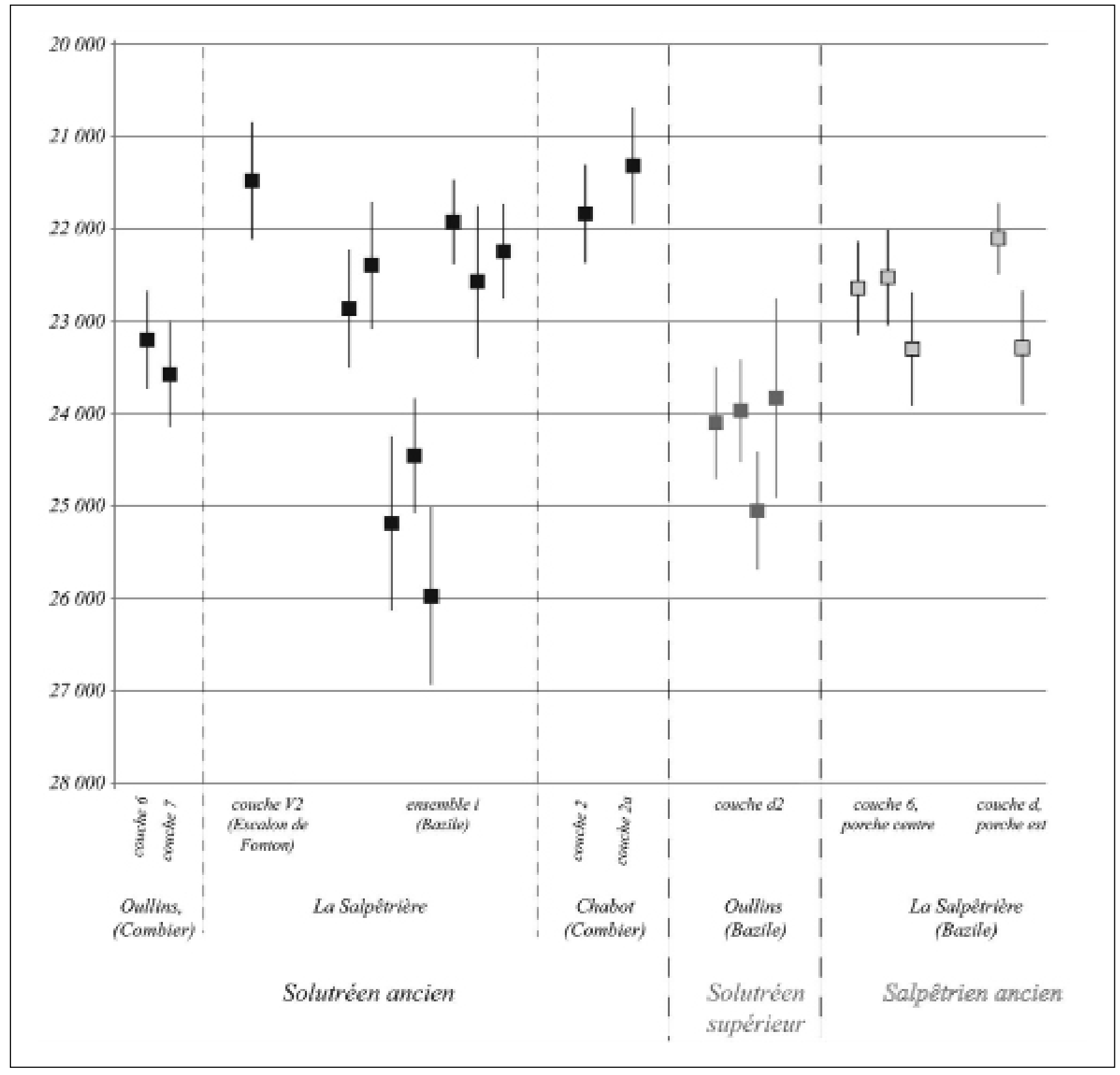

A Figure 3. Datations disponibles calibrées (d'après Bazile, 1990). 
des Grottes Ornées", initié en 2008 par Julien Monney pour les grottes Chabot, Oullins et de la Tête du Lion (Monney et al. à paraître), aurait pu permettre d'éclaircir ce tableau un peu sombre, si les dates obtenues n'étaient pas tout aussi troublantes. En effet, le résultat à Chabot donne une date tout aussi jeune que les précédentes obtenues par J. Combier, autour de 19000 ans BP (Monney et al.à paraître), alors que la stratigraphie montre deux niveaux d'occupation attribués au Solutréen ancien à pointe à face plane et un niveau plus profond paléolithique supérieur indéterminé (Combier 1967). Tandis qu'à Oullins, les nouvelles dates obtenues dans la deuxième salle, avoisinent les 21500 BP (Monney et al.à paraître) pour un sol d'occupation recouvert de calcite, se rapportant au Solutréen récent en raison de la présence en très grand nombre de pointes à cran (Combier 1967).

\section{LE SUJET D'ETUDE}

Dans le cadre de notre thèse, nous avons intégré l'étude du mobilier lithique de quatre gisements, en fonction de la disponibilité des collections archéologiques et de leur relative intégrité stratigraphique : trois gisements solutréens, les gisements d'Oullins, de Chabot et de Granouly, conservés au Musée d'Orgnac-l'Aven, et un gisement épigravettien, le gisement de la Font-Pourquière (Vaucluse, France), conservé au Musée Calvet à Avignon.

\subsection{La grotte d'Oullins}

Le premier site que nous avons étudié est l'important gisement d'Oullins, ou Oulen, situé à cheval entre les départements du Gard et de l'Ardèche, sur la commune de Labastide-de-Virac, sur la rive droite de l'Ardèche, au milieu des gorges.

Connue dès la fin du XIXe siècle, la grotte a longtemps souffert des nombreuses fouilles clandestines (Fig. 4), et il faut attendre la fin des années 1930 avec les fouilles de Maurice Martin pour que les premiers relevés, croquis et rapports soient effectués. Maurice Martin identifie près de la paroi, au fond de la grotte, deux occupations très riches, se rapportant au Solutréen: un premier niveau à pointes à face plane et pointes à cran, qu'il apparente à la phase récente du Solutréen, et un deuxième niveau sous-jacent, à unique pointes à face plane, qu'il apparente à la phase ancienne du Solutréen (Martin 1949).

Dans les années 1950, Jean Combier entreprend des fouilles dans la plupart des gisements ardéchois, dont Oullins. II met au jour plusieurs niveaux stratifiés, allant du

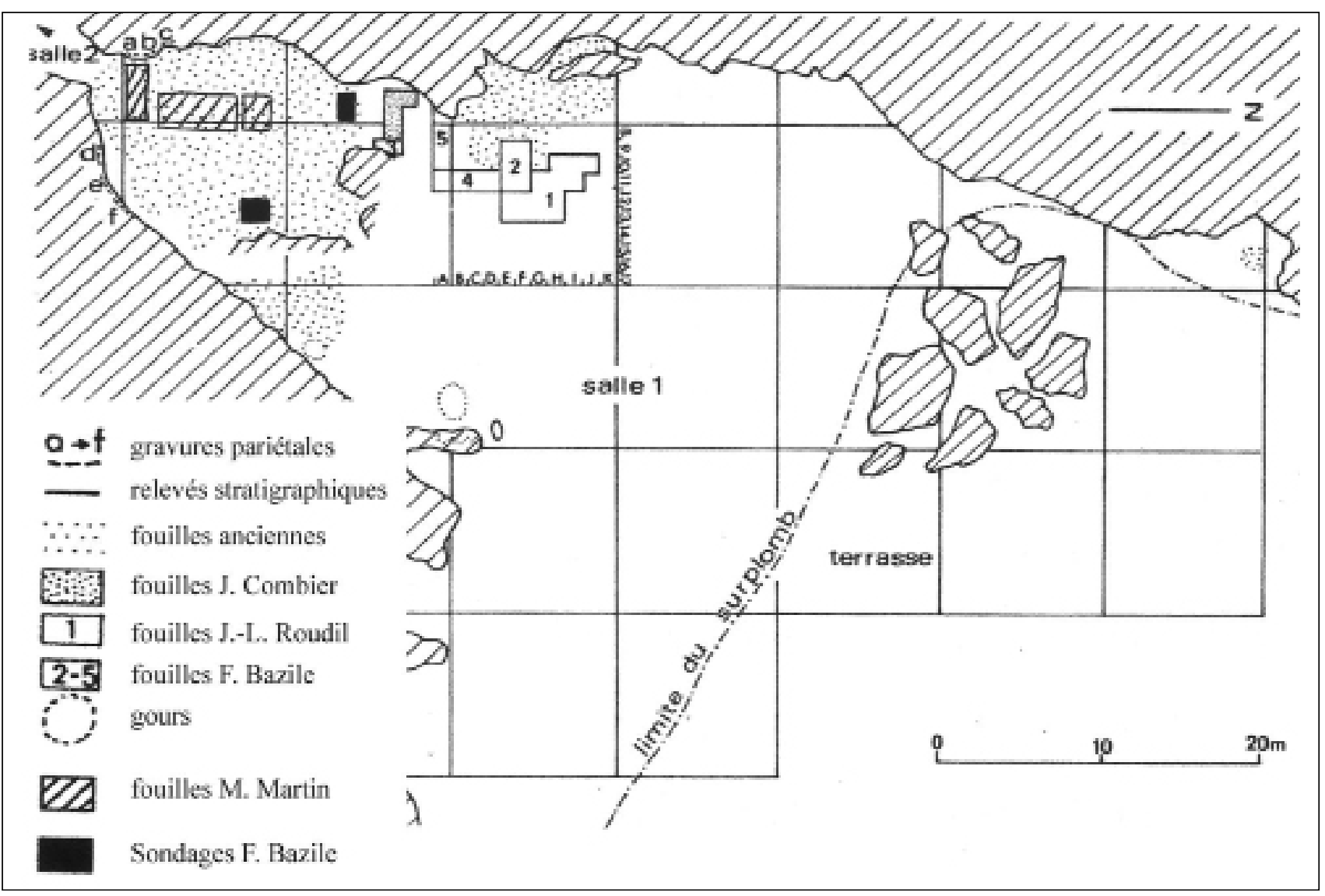

$\Delta$ Figure 4. Plan des différentes fouilles à la grotte d'Oullins (d'après Debard, 1988, modifié). 


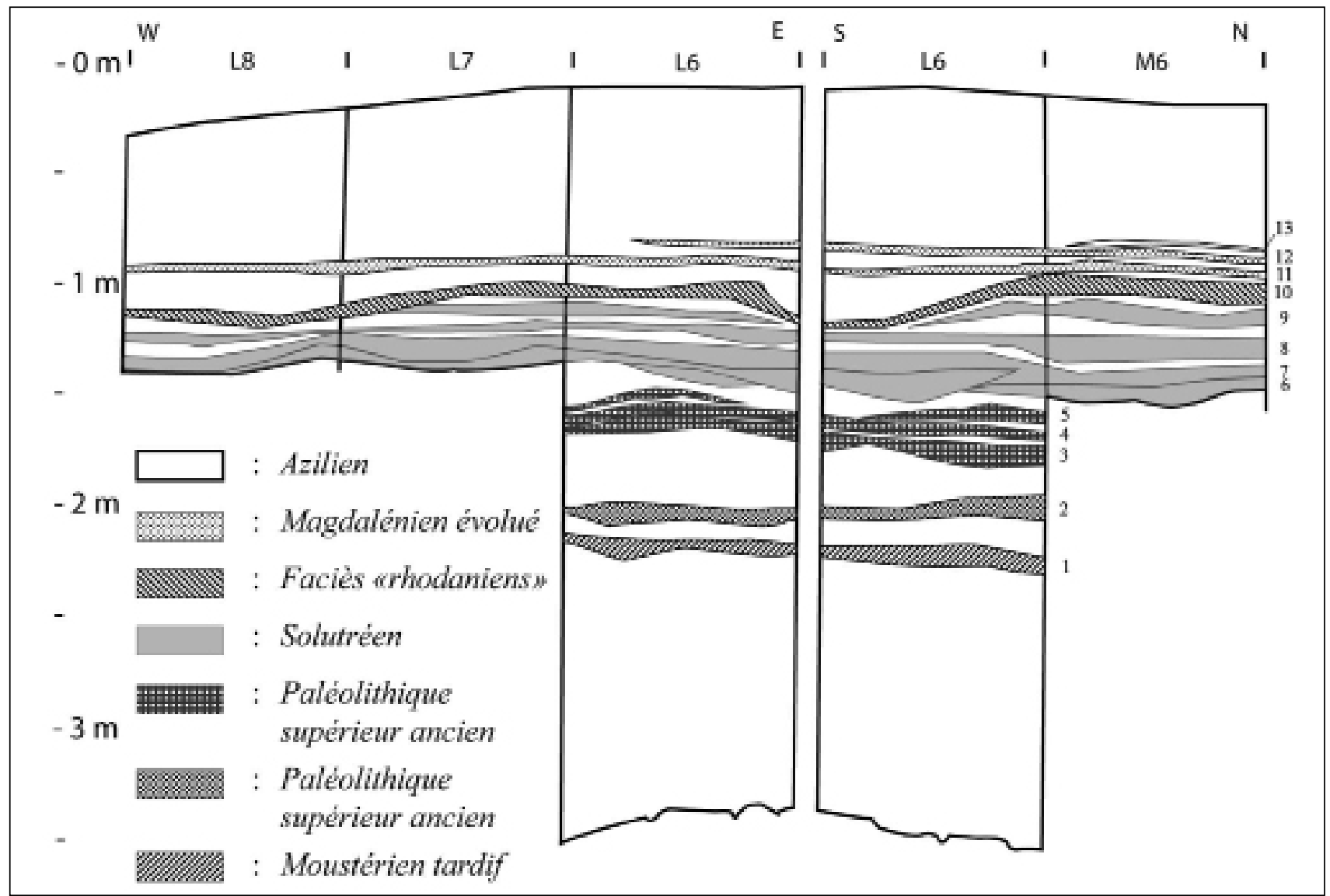

A FiguRE 5. Stratigraphie des fouilles J. Combier à Oullins (d'après Combier, 1967, modifié).

Moustérien jusqu'à I'Azilien, et identifie deux niveaux attribués au Solutréen ancien. Ces deux niveaux, très pauvres en outillage, se caractérisent par un très grand nombre de micro-éclats très souvent indéterminables. Jean Combier lui-même, dans sa thèse, dit ne pas avoir retrouvé les niveaux des fouilles M. Martin (Combier 1967). A la fin des années 1970, c'est au tour de Frédéric Bazile, dans le cadre de fouilles de sauvetage en collaboration avec Jean-Louis Roudil pour la partie Néolithique, d'entreprendre des sondages dans la grotte. Ces recherches sont plus spécifiquement portées sur la phase récente du Solutréen et il y met au jour deux niveaux à pointes à cran (Bazile et BazileRobert 1979).

Nous avons intégré à notre étude les deux collections se rapportant au Solutréen ancien, qui sont les collections Maurice Martin et Jean Combier. La collection Maurice Martin comprend le niveau 2 à pointes à face plane, ayant par ailleurs fait l'objet de deux travaux précédents (Barbiero 2002; Guégan 2007). Bien que manifestement triée et ayant subi une faible contamination du niveau supérieur (quelques pointes à cran et une production de supports laminaires fins et étroits), cette collection reste néanmoins inéluctable pour la richesse des pointes à face plane. En ce qui concerne les fouilles Jean Combier, la phase ancienne du Solutréen est attribuée pour les niveaux 6 et 7. L'intérêt de ces deux séries, bien que assez pauvres, réside dans la composante lamellaire brute et retouchée, qui, associée à un bon contexte stratigraphique, complète les collections anciennes.

\subsection{La grotte Chabot}

La grotte Chabot est située sur la commune d'Aiguèze (Gard), en aval des gorges de l'Ardèche, dans le secteur du Ranc-Pointu, connu pour abriter plusieurs gisements paléolithiques (Moncel 1996).

Comme Oullins, la grotte Chabot est fréquentée depuis au moins la fin du XIXe siècle et a fait l'objet de nombreuses campagnes de fouilles clandestines. En 1872, Léopold Chiron, instituteur local, a identifié des gravures qu'il ne signala qu'en 1878 (Combier 1997; Goury 1997). Mais il lui faudra attendre la découverte d'Altamira en 1895, et les épisodes que l'on connaît, pour que sa découverte soit authentifiée et approuvée (Combier 1997; Goury 1997).

Plusieurs campagnes se sont succédées à la grotte Chabot dans la première moitié du XXe siècle, mettant au jour, à chaque fois, un seul niveau de solutréen ancien à pointes à face plane. Jean Combier procéda à plusieurs sondages dans la grotte à la fin des années 1950, où il 


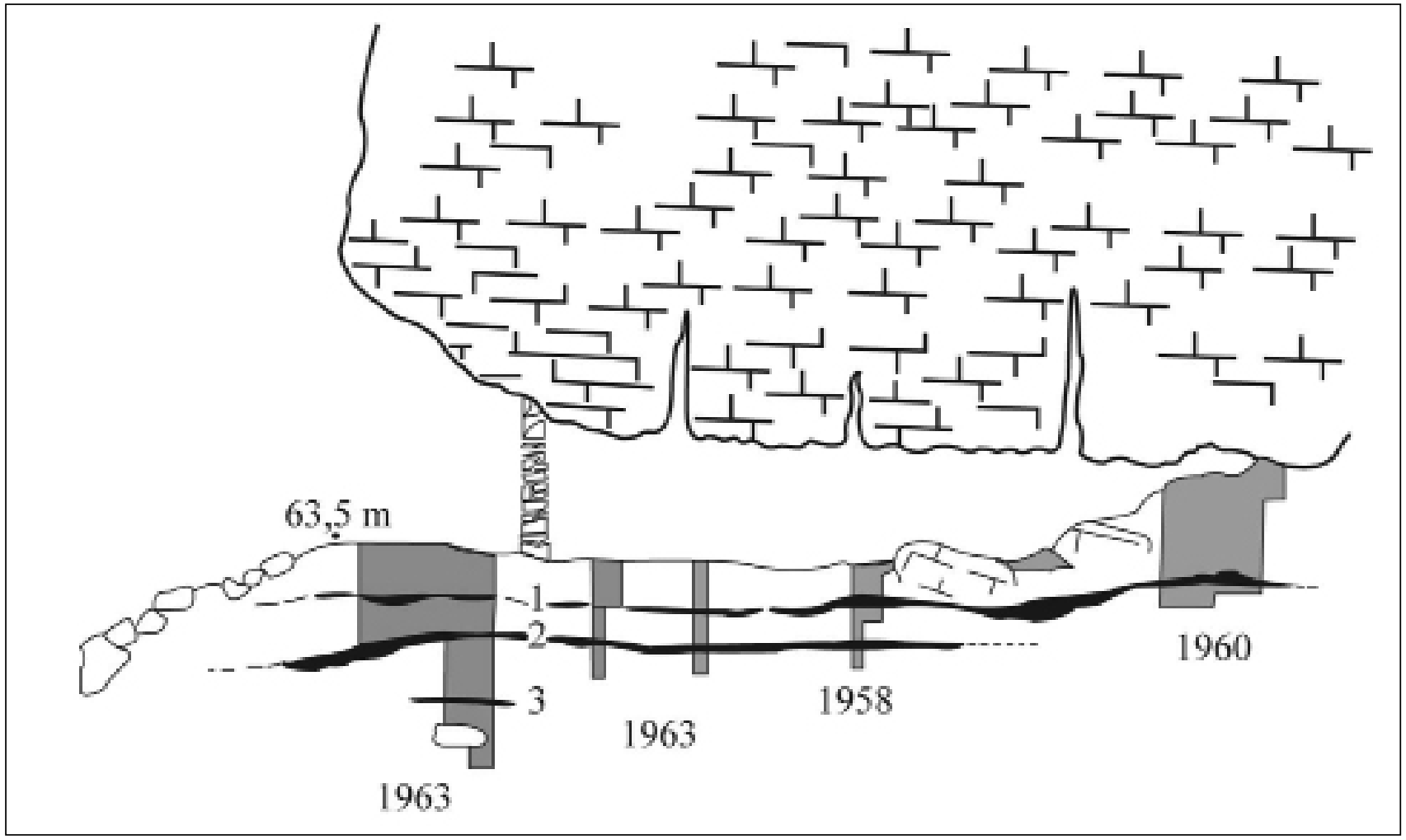

A Figure 6. Plan schématique de la grotte Chabot et chronologie des fouilles J. Combier (d'après Combier, 1967, modifié).

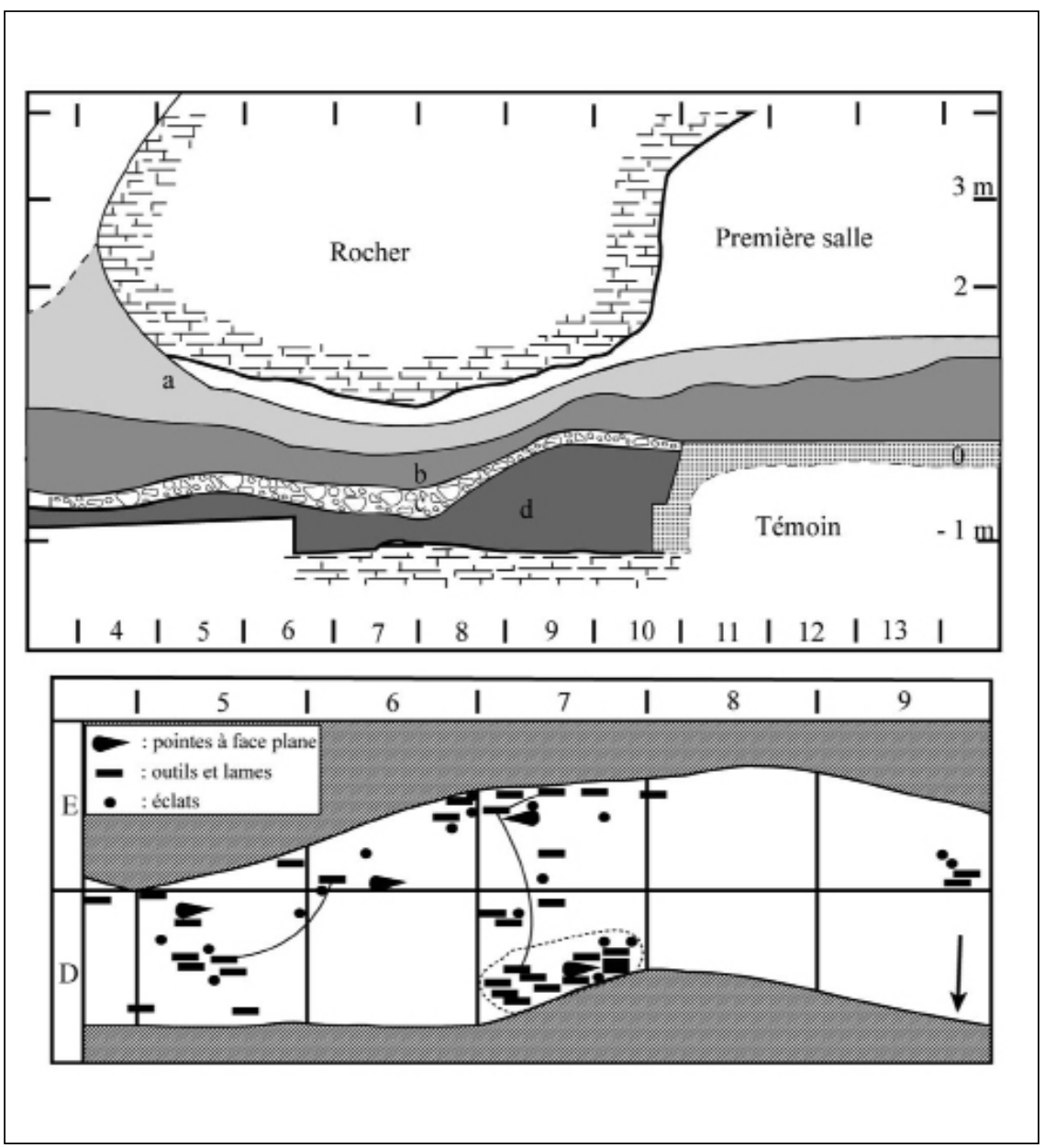

4 FiguRe 7. Plan schématique de la grotte de Granouly et distribution spatiale du mobilier lithique (extrait de Combier, 1973, modifé). 


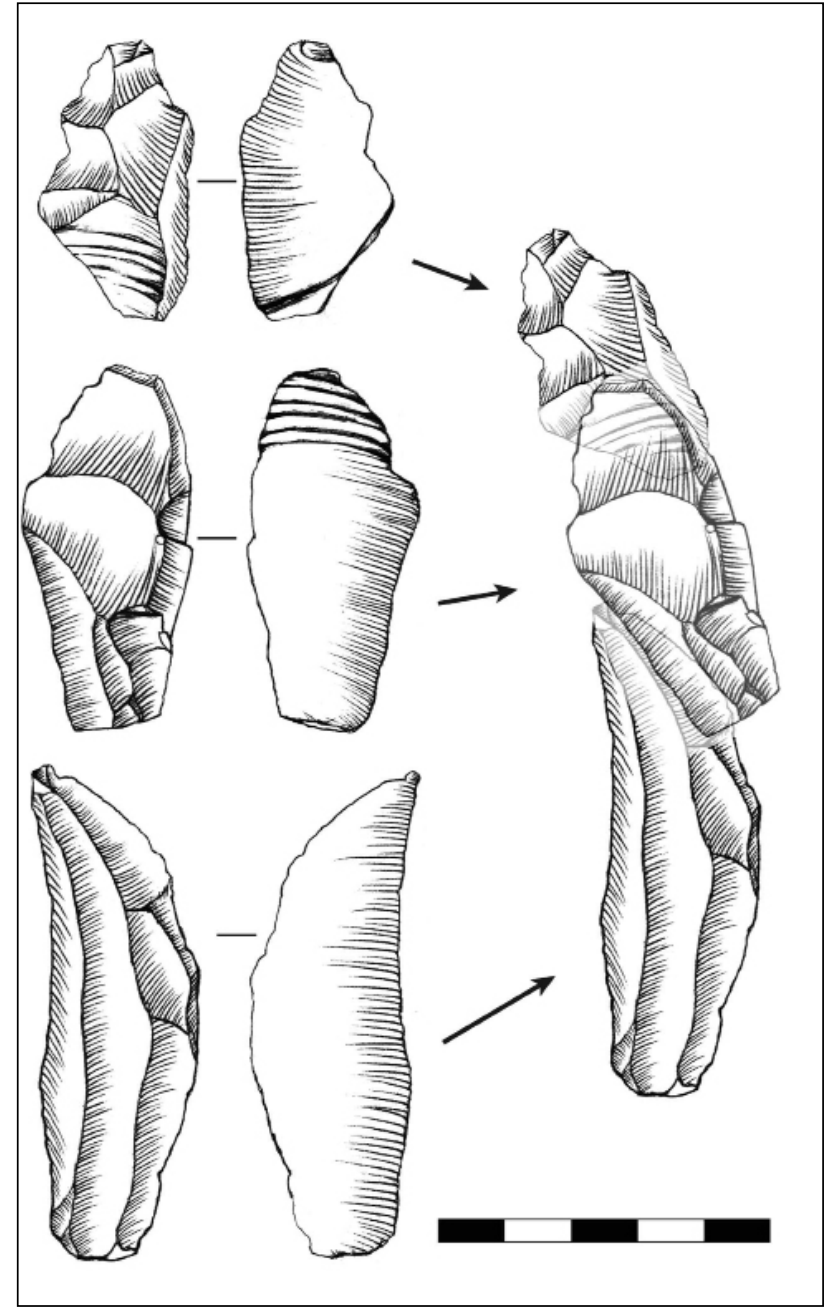

$\Delta$ FIgURE 8. Remontage effectué entre trois produits d'entretien des flancs à la grotte de Granouly (dessins Sophie Guégan).

retrouva ce niveau, auquel s'ajoute un deuxième niveau sous-jacent, attribué également à la phase ancienne du solutréen (Fig. 6), et dont il ne dit avoir fouillé que le sommet (Combier 1967).

II effectue enfin un dernier sondage, à l'entrée de la grotte, au début des années 1960, plus profond, dans lequel il trouve, à un mètre sous le second niveau solutréen, un niveau Paléolithique supérieur indéterminé (Combier 1967).

La grotte Chabot fait donc parti de cette liste trop longue de sites fouillés trop tôt, entrainant le morcellement des différentes collections qui en sont issues et donc la perte d'informations. Plusieurs collections sont donc conservées au musée d'Orgnac-l'Aven (Ardèche), réunissant les différents ramassages de surface et les séries issues des diverses campagnes de fouilles, qui, pour ces dernières, sont malheureusement incomplètes. Du reste, avec ces deux niveaux de Solutréen ancien à pointes à face plane, la grotte Chabot demeure un site incontournable pour comprendre l'évolution de cette phase en Ardèche.

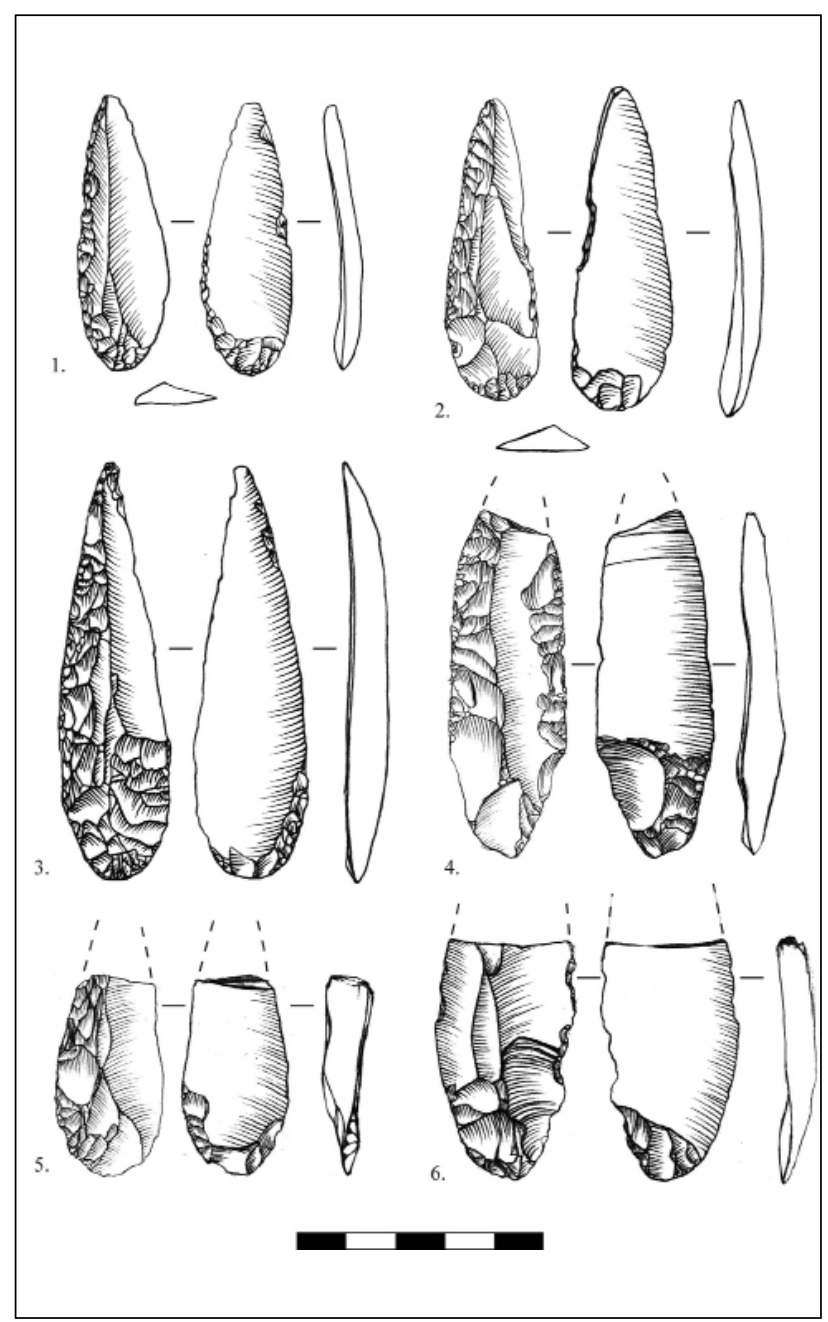

$\Delta$ Figure 9. Pointes à face plane. 1 à 3: Oullins, niveau 2, fouilles M. Martin; 4 à 6: Granouly. (dessins Sophie Guégan).

\subsection{La grotte de Granouly}

Contrairement aux précédentes, la grotte de Granouly n'est pas située dans les gorges, mais sur la commune du Pouzin, à une quarantiane de kilomètres au nord.

Cette petite grotte s'est formée dans un massif calcaire, sous le Plateau des Gras, à quelques centaines de mètres des rives du Rhône, et non loin de son affluent, l'Ouvèze. Soulignons qu'au Pléniglaciaire supérieur, et au niveau de la commune du Pouzin en particulier, le Rhône, plus étroit, coulait légèrement plus à l'Est, à un kilomètre environ de la rive droite actuelle (Mandier 1988).

La grotte, toujours en activité, est une cavité étroite, d'à peine un mètre de large, pour une hauteur d'1m50 environ. Elle est constituée de plusieurs salles difficiles d'accès. La fouille a eu lieu au début des années 1960, à l'entrée de la grotte et dans son prolongement, dont il reste un témoin conservé avec de la faune et des charbons (Fig. 7).

La collection récoltée lors des fouilles est composée de 115 pièces lithiques dont 4 pointes à face plane et quelques 


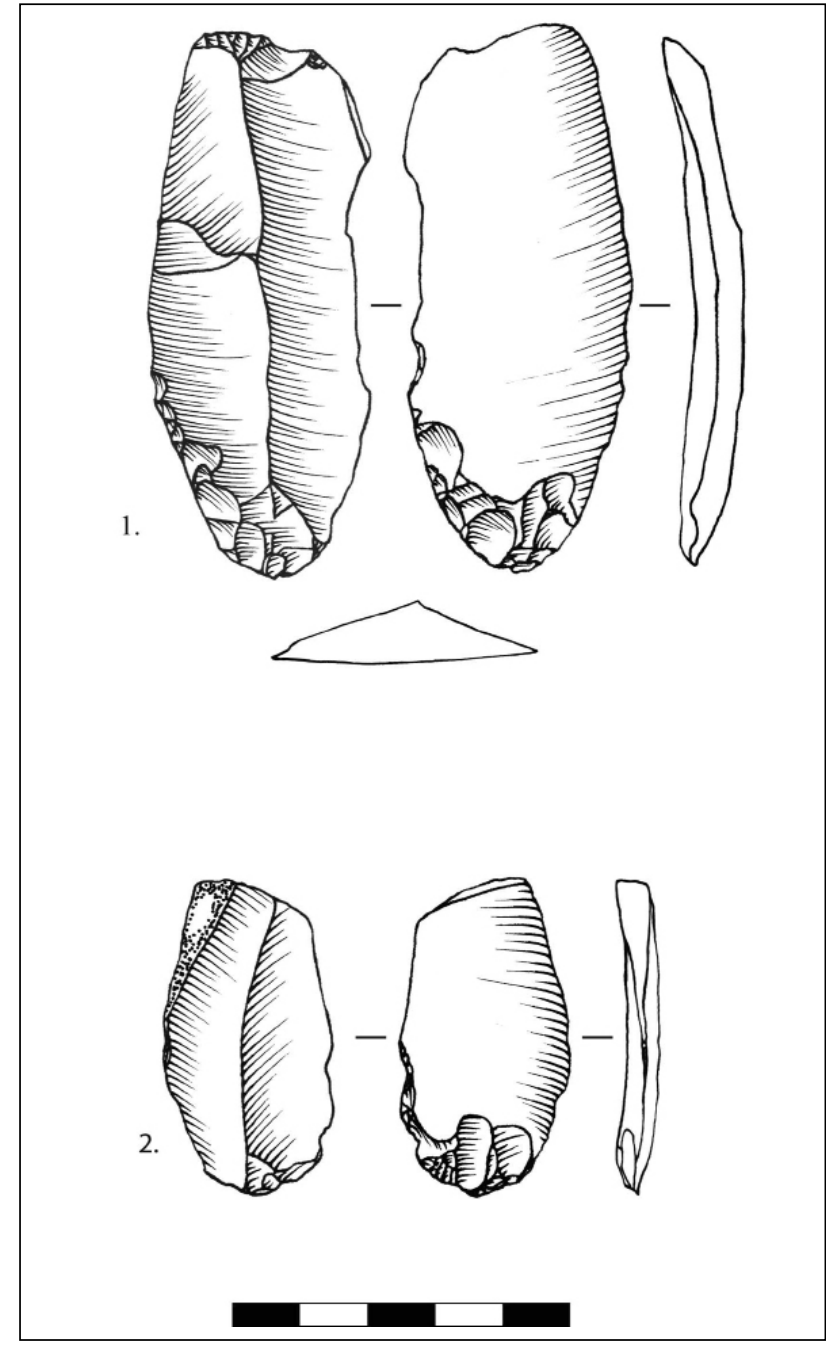

\ Figure 10. Ebauches de pointes à face plane, Oullins, niveau 2 des fouilles M. Martin (Dessins Sophie Guégan).

restes de faunes assez mal conservés (Combier 1973). Aucun nucléus n'est présent dans cette série, mais quelques remontages ont pu être effectués (Fig. 8).

\section{OBSERVATIONS PRÉLIMINAIRES}

\subsection{L'outillage}

Nous avons principalement axé notre étude sur de la pointe à face plane, objet emblématique et particulièrement bien représentée dans les collections ardéchoises. Elle comporte presque toujours une base arrondie à l'aide d'une retouche plate et rasante sur les deux faces, qui peutêtre envahissante à couvrante (Fig. 9, n. 1 à 3 et n. ${ }^{\circ}$ ). La retouche progresse toujours de manière continue sur le bord gauche et sur la face supérieure uniquement, devenant ainsi le plus souvent semi-abrupte en partie apicale. Le bord droit est, dans la majorité des cas, laissé brut. Les quelques va-

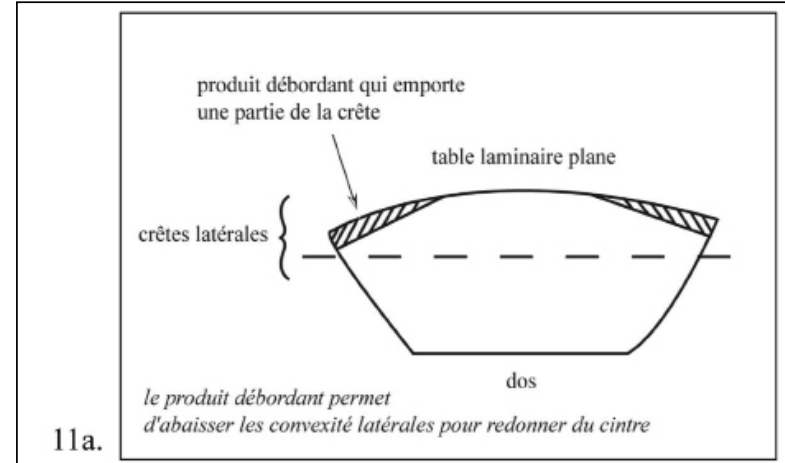

$11 b$.

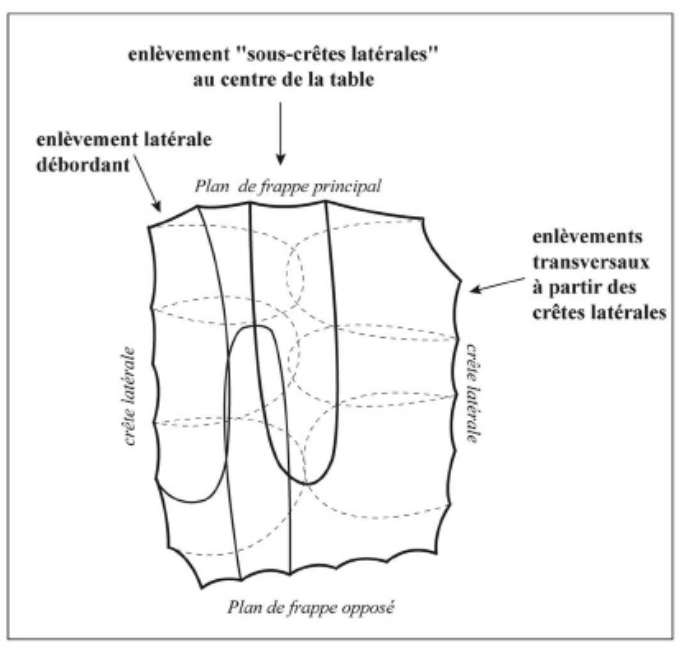

\ FIguRE 11a. Schéma de l'entretien des convexités (DAO Sophie Guégan).

\ FiguRE 11b. Schéma du débitage laminaire (DAO Sophie Guégan)

riantes concernent d'une part la pointe, qui peut être axiale ou déjetée vers la droite, et d'autre part l'orientation technologique de la pièce, certaines pièces ont en effet leur base aménagée en partie distale de la lame et leur partie apicale aménagée en partie proximale (Fig. 9 n. ${ }^{\circ}$ ), alors que le plus fréquemment, la base arrondie se trouve en partie proximale et l'apex en partie distale, de façon à amincir la pièce et régulariser le profil.

Concernant la fonctionalité des pointes à face plane, la seule étude tracéologique entreprise est celle menée par Hugues Plisson sur les pointes à face plane du niveau 6 d'Azkonzilo (Pyrénes-Atlantique) dans le cadre de la thèse de Caroline Renard (Renard 2010). Les_résultats montrent un usage différent entre les pointes axiales, dont l'emmanchement s'effectue dans l'axe de la pièce, pour une utilisation de la pointe et des bords tranchants et les pointes déjetées, dont l'emmanchement se fait latéralement, pour un emploi du bord tranchant laissé libre (Renard 2010 : 133). 


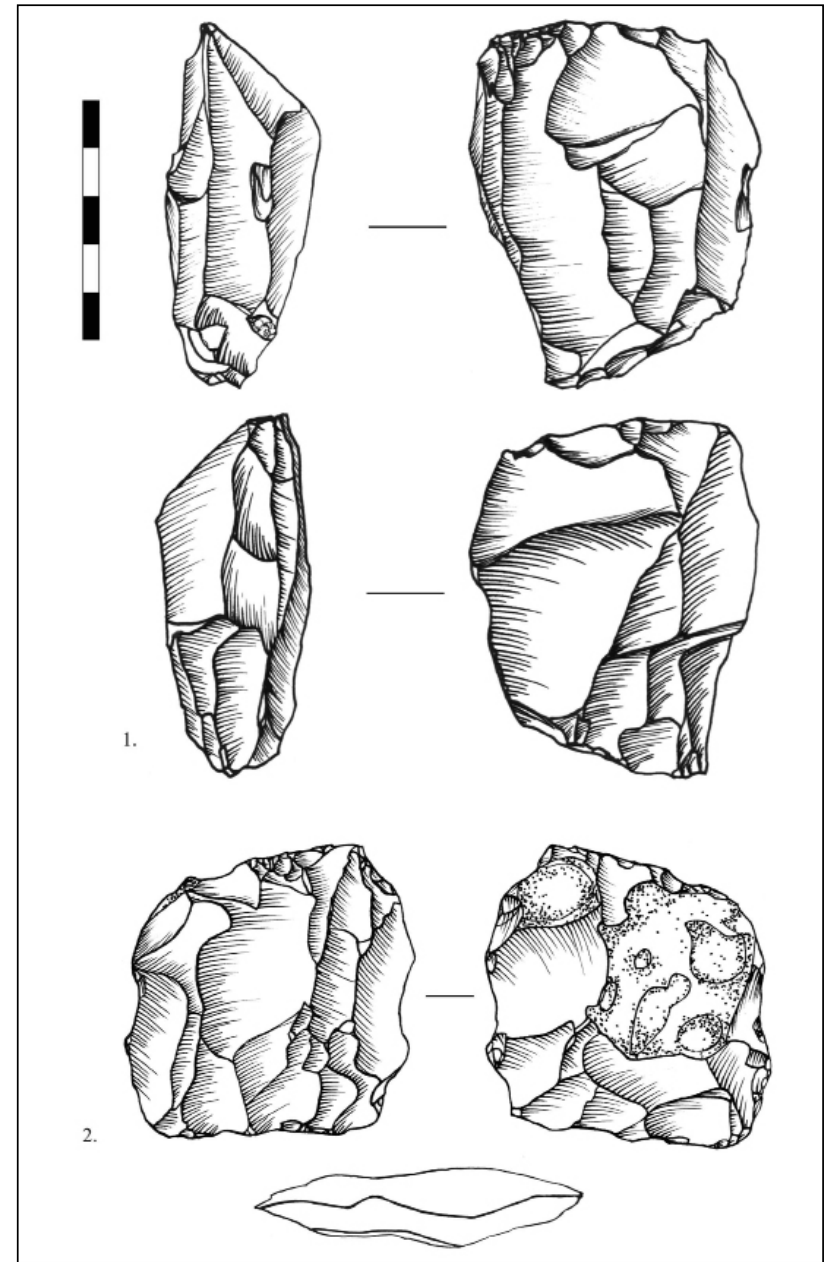

A Figure 12. Nucléus à lames. 1: grotte Chabot, issu des ramassages de surface; 2: Oullins niveau 2 des fouilles $\mathrm{M}$. Martin (Dessins Sophie Guégan).

\subsection{Le débitage}

L'observation technologique des nucléus et de l'ensemble des produits montre que le débitage est orienté vers la production de supports laminaires larges, destinés à être les futurs supports des pointes à face plane (Fig. 10). La mise en place de crêtes latérales permet de débiter des enlèvements transversaux couvrants, préparant la future surface de débitage (Fig. 11a), tout en maintenant une certaine amplitude à la table. L'exploitation laminaire est majoritairement unipolaire, mais on peut noter la présence assez fréquente d'un second plan de frappe opposé qui est une alternative contribuant à entretenir la surface de débitage tout en maintenant les convexités (Fig. 11b). Ce schéma, observé dans l'ensemble des collections que nous avons étudiées, permet d'établir une réelle cohérence entre les ensembles, malgré les problèmes de tri, d'ancienneté des fouilles et les probables contaminations, évoqués plus haut (Fig. 12).

Ce schéma de débitage, singulier au Paléolithique supérieur, a été identifié en premier dans le Bassin parisien par Caroline Renard (Renard 2002), et on le retrouve qui perdure dans les phases récentes, comme dans la couche 21 de Laugerie-Haute Est, attribuée au Solutréen final (Renard 2010).

\subsection{Les matières premières}

Si les collections de Chabot et de Granouly n'offrent pas la possibilité de déterminer les matières premières utilisées, en raison de la patine qui couvre l'ensemble des pièces lithiques, à Oullins, en revanche la bonne conservation du silex et sa grande diversité permettent d'établir une carte de déplacements tout à fait originaux pour le Maximum Glaciaire, dont nous présentons ici les grandes lignes (Fig. 13).

Si les matières premières très locales, comme le Ludien de Laval-Saint-Roman ou I'Oligocène d'Orgnac-l'Aven ont été utilisées par les Solutréens d'Oullins, la matière la plus abondamment utilisée (près de $70 \%$ de la collection) est un silex du Bédoulien d'excellente qualité, dont on localise les gîtes à une trentaine de kilomètres au nord des gorges, à Meysse-Rochemaure (Ardèche).

Une dizaine de produits bruts et retouchés, dont un grattoir sur éclat portant une large plage semi-corticale crayeuse sur la face supérieure, ont été obtenus dans un silex blond très translucide et à la texture très fine. L'examen de ces pièces par Javier Mangado a permis d'envisager une origine turonnienne de la vallée du Cher de ces silex, et plus particulièrement le type C3a-2 de Thierry Aubry (Aubry 1991), établissant ainsi un déplacement sur presque $500 \mathrm{~km}$. II est intéressant de noter que cette matière identifiée à Oullins, se retrouve à la fois dans les niveaux attribués au Solutréen ancien et dans ceux attribués au Solutréen récent (Boocaccio 2005), dans les vieilles collections comme les récentes. Par ailleurs, le Turonien inférieur du Cher est une matière que l'on retrouve aussi bien dans certains gisements du Gravettien tels que la Vigne-Brun dans le département de la Loire (Masson 1981; Pesesse 2008) ou dans certains gisements du Magdalénien du Sud de la France tels que la grotte Gazel (Langlais 2007), donnant des distances équivalentes à celle qui séparerait la baume d'Oullins de la vallée du Cher. Néanmoins, et dans l'attente de proposition alternative, nous gardons une certaine réserve quant à ces résultats qui bouleversent certains à priori concernant à la fois les déplacements sur de grandes distances au Paléolithique, mais aussi les modes de vie des Hommes Préhistoriques durant le Maximum Glaciaire.

Enfin, et non des moindres, la présence de quelques pièces retouchées en silex du Barrémien de Maloubret, autrement dit directement sur la rive gauche du Rhône (non loin de la grotte Mandrin) et un grattoir en silex du Barrémo-Bédoulien du Mont-Ventoux, très caractéristique avec sa couleur bleue-grise et son cortex jaune-orangé, atteste un franchissement du Rhône. 


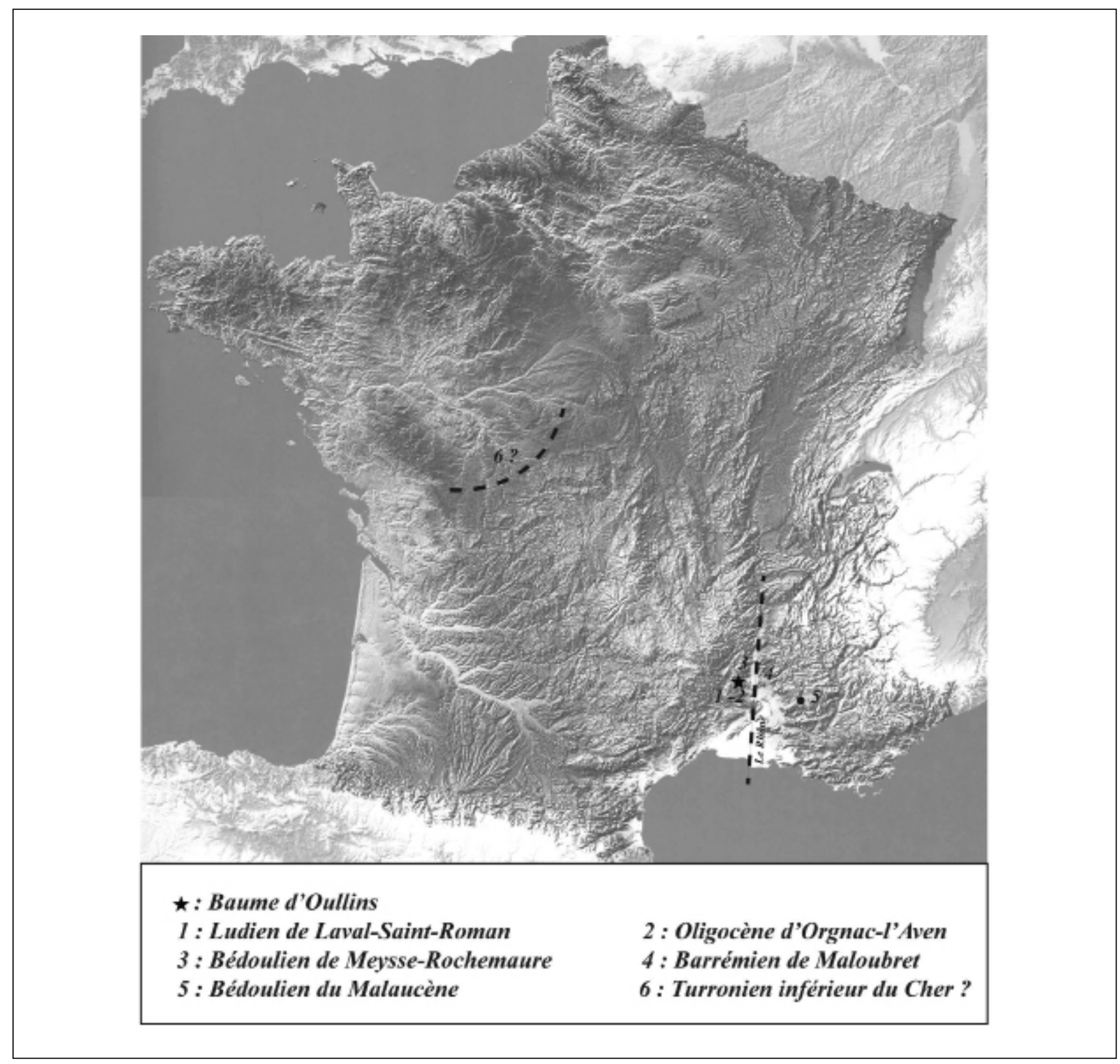

\ Figure 13. Carte de diffusion des principales matières premières présentes sur le gisement d'Oullins (DAO Sophie Guégan).

\section{CONSIDÉRATIONS FINALES}

De tels résultats offrent des pistes de réflexions encourageantes concernant les problématiques de contacts, d'échanges et de probables diffusions d'idées, entre les deux rives du fleuve, qui ont donc été franchies. Les deux rives du Rhône sont, en effet, à ce moment précis du Pléniglaciaire supérieur, occupées par deux groupes de populations d'Hommes aux techniques à priori différentes: les Solutréens sur la rive droite et les Epigravettiens sur la rive gauche. L'étude du gisement de la Font-Pourquière, situé non loin du Mont Ventoux, entre Apt et Cavaillon, dans le Vaucluse, devrait apporter des éléments de réponse. Ce gisement, qui est le point le plus occidental de la sphère épigravettienne, mais qui est également le plus proche de la sphère solutréenne, est attribué à l'Epigravettien ancien à "pointes à face plane", autrement dit, la première phase de ce vaste techno-complexe, sans pointes à cran. Il est difficile à ce jour, étant donné les problèmes de chronologie déjà évoqués plus haut, de statuer sur une contemporanéité directe avec les Solutréens d'Ardèche, d'autant que les datations obtenues sur les niveau épigravettiens anciens, sont tout, elles-aussi, problématiques (Onoratini 1982). Néanmoins, la situation géographique de ce site ainsi que sa chronologie relative, font de lui un témoin privilégié pour discuter de l'occupation de ce territoire au Pléniglaciaire supérieur. 


\section{REMERCIEMENTS}

Je souhaiterais remercier en premier lieu mon directeur de thèse, Frédéric Bazile, pour m'avoir confié la collection $M$. Martin à la Baume d'Oullins. Je tiens également à remercier toute l'équipe du Musée d'Orgnac-l'Aven et principalement Sonia Stocchetti et Nicolas Lateur, ainsi que Monsieur Jean
Combier et Julien Monney. Je souhaite également remercier le musée Calvet qui m'a confié la série de la Font-Pourquière, et je tiens à exprimer ma gratitude envers Guillaume Boccaccio, Céline Bressy, Ludovik Slimak et Damien Pesesse pour les discussions et le temps passé sur les matières premières. Enfin je tiens à exprimer toute ma reconnaissance à Damien Flas et Maria Lorenzo Martinez pour leurs traductions.

\section{BIBLIOGRAPHIE}

AUBRY, T. 1991: L'exploitation des ressources en matières premières lithiques dans les gisements solutréens et badegouliens du bassin versant de la Creuse (France). Thèse de doctorat de I'Université de Bordeaux I. Bordeaux.

BARBIERO, A. 2002: Analyse typo-technologique des pointes à face plane et des pointes à cran de la Baume d'Oullins (Labastide-de-Virac, Ardèche, le Garn, Gard). Mémoire de Maîtrise, Université de Paris 1 Panthéon-Sorbonne. Paris.

BAZILE, F. et BAZILE-RobeRT, E. 1979: "Le Solutréen à pointe à cran de la Baume d'Oullins (Le Garn, Gard et Labastide-de-Virac, Ardèche) Position chronostratigraphique". Etudes Préhistoriques 15: 1-6.

BAzILE, F. 1990: "Le Solutréen et l'Episolutréen dans le Sud-Est de la France, in : Les industries à pointes foliacées du Paléolithique supérieur européen". En J.K. Kozlowski (dir.): Feuilles de pierre. Les industries à pointes foliases du Paléolithique supériur européen. Actes $d u$ Colloque de Cracovie. ERAUL 42: 393-424.

BAZILE, F. et BocCACCIO, G. 2007: Du "Solutréen supérieur au Magdalénien en Languedoc rhodanien : ruptures et continuités". Bulletin de la Société préhistorique française 104: 787-796.

- 2008: "Le Salpêtrien, un technocomplexe épisolutréen redéfini". Gallia Préhistoire 50: 103-141.

Boccaccio, G. 2005: Les industries lithiques du Solutréen supérieur et du Salpêtrien ancien en Languedoc : ruptures et continuités des traditions techniques. Thèse de Doctorat de I'Université Aix-Marseille I.

Combier, J. 1967: Le Paléolithique de l'Ardèche dans son cadre paléoclimatique. Impr. Delmas. Bordeaux.

- 1973: L'industrie solutréenne de deux nouveaux sites de l'Ardèche, Etudes préhistoriques 5: 1-7.

- 1997: L'origine des recherches sur la Préhistoire ancienne de I'Ardèche, Mémoire d'Ardèche et Temps Présents, tome 56, Association Mémoire d'Ardèche et Temps Présent, Privas : 7-20.

DeBARD, E. 1988: Le Quaternaire du Bas-Vivarais d'après l'étude des remplissages d'avens, de porches et d'abri sous-roche. Dynamique sédimentaire, paléoécologique et chronologique. Documents des Laboratoires de Géologie de Lyon 103. Lyon.

DemarS, P. Y., 1995: "Le Solutréen de Laugerie-Haute (Dordogne). Economie du silex". Gallia Préhistoire 37: 1-53.

Escalon De Fonton, M. et Bonifay, E. 1957: "Les niveaux solutréens de la grotte de la Salpêtrière". L'Anthropologie 61 (3-4): 207-238.

GELY, B. 2008: "Les premiers peuplements en bas-vivarais". En M. Raimbault, C. Rouchouse et H. Ozil (eds.): De la Dent de Rez au Gorges de l'Ardèche. Histoire et environnement d'un territoire. Éditions de l'lbie, 128-137.
GoURY, J. 1997: "Léopold Chiron (1845-1916), l'instituteur archéologue". Mémoire d'Ardèche et Temps Présents, tome 56, Association Mémoire d'Ardèche et Temps Présent. Privas: 45-50.

GuÉGAN, S. 2007: Le Solutréen ancien en moyenne vallée du Rhône. Réflexion à partir du matériel lithique de la Baume d'Oullins (GardArdèche, France). Mémoire de Master 2, Université de Paris 1 Panthéon-Sorbonne. Paris.

LANGLAIS, M. 2007: Dynamiques culturelles des sociétés magdaléniennes dans leurs cadres environnementaux. Enquête sur 7000 ans d'évolution de leurs industries lithiques entre Rhône et Ebre. Thèse de Doctorat de I'Université de Toulouse - Le Mirail.

MANDIER, P. 1988: Le Relief de la moyenne vallée du Rhône au Tertiaire et au Quaternaire: Essai de synthèse paléogéographique. Documents du BRGM 151.

MARTIN, M. 1949: "Le Protosolutréen dans le Gard". Bulletin de la Société préhistorique française 46: 10-12.

MASSON, A. 1981: Pétroarchéologie des roches siliceuses. Intérêt en Préhistoire. Thèse de Doctorat de I'Université Lyon I. Lyon.

Moncel, M.-H. 1996: "Le Moustérien de la grotte du Ranc Pointu (Ardèche)". Bulletin de la Société Préhistorique Française 93: 164168.

Monney, J., Combier, J., Kaltnecker, E., Lateur, N., Stocchetti, S. et VallaDAS, H. À PARAîTRE: "Nouveaux éléments de discussion chronologiques dans le paysages des Grottes ornées de l'Ardèche: Oulen, Chabot et la Tête-du-Lion". Paléo hors série à paraître.

OnORATINI, G. 1982: Préhistoire, sédiments et climats du Würm III à I'Holocène dans le Sud-Est de la France. Travaux E. R. 46, Université d'Aix-Marseille III.

PESESSE, D. 2008: Les premières sociétés gravettiennes. Analyse comparée des systèmes lithiques de la fin de l'Aurignacien aux débuts $d u$ Gravettien. Thèse de Doctorat de I'Université de Provence.

Prud'Homme, F. 2008: "Les grottes ornées des gorges de l'Ardèche". En M. Raimbault, C. Rouchouse et H. Ozil (eds.): De la Dent de Rez au Gorges de l'Ardèche. Histoire et environnement d'un territoire. Éditions de l'Ibie: 148-158.

RENARD, C. 2002: "Des témoins solutréens en France septentrionale : un mode original de production de support de pointe à face plane (La Celle-Saint-Cyr, Yonne)". Bulletin de la Société préhistorique française 99: 461-485.

- 2010: Les premières expressions du Solutréen dans le Sud-Ouest français. Evolution techno-économique des équipements lithiques au cours du dernier maximum glaciaire. BAR International Series 2070. Oxford.

SмITH, P.E.L. 1966: Le Solutréen en France. Impr. Delmas. Bordeaux. 